\title{
Microscopic description of Coulomb and nuclear excitation of multiphonon states in ${ }^{40} \mathrm{Ca}+{ }^{40} \mathrm{Ca}$ collisions
}

\author{
M. V. Andrés \\ Departamento de Física Atómica, Molecular y Nuclear, Universidad de Sevilla, Apdo 1065, E-41080 Sevilla, Spain \\ F. Catara and E. G. Lanza \\ Dipartimento di Fisica Universitá di Catania and INFN, Sezione di Catania, I-95129 Catania, Italy \\ Ph. Chomaz \\ GANIL, Boîte Postale 5027, F-14021 Caen Cedex, France
}

M. Fallot and J. A. Scarpaci

Institut de Physique Nucléaire, IN2P3-CNRS, F-91406 Orsay Cedex, France

(Received 2 January 2001; published 14 December 2001)

\begin{abstract}
We calculate the inelastic scattering cross sections to populate one- and two-phonon states in heavy ion collisions with both Coulomb and nuclear excitations. Starting from a microscopic approach based on randomphase approximation, we go beyond it in order to treat anharmonicities and nonlinear terms in the exciting field. These anharmonicities and nonlinearities are shown to have important effects on the cross sections both in the low energy part of the spectrum and in the energy region of the double giant quadrupole resonance. By properly introducing an optical potential, the inelastic cross section is calculated semiclassically by integrating the excitation probability over all impact parameters. A satisfactory agreement with the experimental results is obtained.
\end{abstract}

DOI: 10.1103/PhysRevC.65.014608

PACS number(s): 25.70.De, 21.60.Ev, 21.60.Jz, 24.30.Cz

\section{INTRODUCTION}

All theoretical approaches used to calculate the cross section for the multiple excitation of giant resonances (GR) in heavy ion collisions are based on a semiclassical description of the process [1] where the excitation of one reaction partner is assumed to be due to the action of the mean field of the other and is treated quantum mechanically while the relative motion is determined classically.

For each eigenstate $\alpha$ of the internal Hamiltonian of one nucleus, one can calculate its excitation probability $P_{\alpha}(b)$ by perturbation theory or by solving a system of coupled equations. This is done by integrating the equations of motion along the classical relative motion trajectory corresponding to the impact parameter $b$. The total excitation cross section $\sigma_{\alpha}$ is then evaluated by integrating the probability over all the impact parameters starting from a minimum one, $b_{\min }$. In Coulomb excitation studies, the value of the latter is chosen according to a systematics [2] following some prescription based on the condition that the contributions from the nuclear field should be eliminated. Even so, however, some ambiguities are present since the calculated cross sections can vary appreciably for small variations of $b_{\min }$. Moreover, when the bombarding energy is not very high and the two nuclei are not very heavy, the nuclear excitation is the dominant process. In this situation one cannot apply that procedure because, in principle, one should add more internal trajectory for the determination of $\sigma_{\alpha}$. On the other hand, the trajectories corresponding to small impact parameters would not contribute too much to the inelastic cross section if the absorption due to all other channels is taken into account.
This can be done by introducing an optical potential as was already done in a qualitative way in Ref. [3].

In this paper we present calculations for the excitation cross section of one- and two-phonon states in the ${ }^{40} \mathrm{Ca}+{ }^{40} \mathrm{Ca}$ reaction at $50 \mathrm{MeV} / \mathrm{m}$ for which experimental results exist [4]. The calculations are done within the extended random-phase approximation (RPA) model described in our previous works [5-7] where we have introduced anharmonicities in the internal Hamiltonian and nonlinear terms in the external field. This model has been successful in the description of the excitation of the double giant resonances (DGR), reducing the discrepancy between the measured cross section and the standard theoretical estimate. Here the model is extended by introducing an optical potential in order to avoid the uncertainty on the integration over the impact parameter. Since the optical potential takes into account the absorption due to all channels, we have introduced a procedure in order to avoid double counting the effects of the channels explicitly included in our calculations. In the following section we will recall briefly our model and extensively describe its improvements. In Sec. III we present our results and the quantitative comparison with the experimental findings. We then draw our conclusions and discuss some perspectives.

\section{THE MODEL}

The best microscopic theory to describe collective excitations in nuclei is the RPA whose Hamiltonian can be written as 


$$
H_{\mathrm{RPA}}=\sum_{\nu} E_{\nu} Q_{\nu}^{\dagger} Q_{\nu}
$$

where the phonon creation operator is

$$
Q_{\nu}^{\dagger}=\sum_{p, h}\left(X_{p h}^{\nu} B_{p h}^{\dagger}-Y_{p h}^{\nu} B_{p h}\right)
$$

The bosonic operators $B$ are the lowest-order terms of the bosonic expansion of the fermionic operators [8]

$$
a_{p}^{\dagger} a_{h} \rightarrow B_{p h}^{\dagger}+(1-\sqrt{2}) \sum_{p^{\prime} h^{\prime}} B_{p^{\prime} h^{\prime}}^{\dagger} B_{p^{\prime} h^{\prime}}^{\dagger} B_{p h^{\prime}}+\cdots .
$$

Here, the index $p(h)$ labels the particle (hole) states with respect to the Hartree-Fock (HF) ground state. The other terms after the first one correct for the Pauli principle.

In the harmonic RPA Hamiltonian (2.1) only the $V_{p h, p^{\prime} h^{\prime}}$ and $V_{p p^{\prime}, h h^{\prime}}$ terms of the residual interaction are taken into

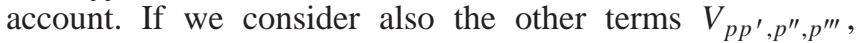
$V_{h h^{\prime}, h^{\prime \prime} h^{\prime \prime \prime}}, V_{p p^{\prime}, p^{\prime \prime} h}$, and $V_{p h, h^{\prime}, h^{\prime \prime}}$ and introduce the mappings [8]

$$
\begin{aligned}
& a_{p}^{\dagger} a_{p^{\prime}} \rightarrow\left(a_{p}^{\dagger} a_{p^{\prime}}\right)_{B}=\sum_{h} B_{p h^{\prime}}^{\dagger} B_{p^{\prime} h}, \\
& a_{h} a_{h^{\prime}}^{\dagger} \rightarrow\left(a_{h} a_{h^{\prime}}^{\dagger}\right)_{B}=\sum_{p} B_{p h^{\prime}}^{\dagger} B_{p h^{\prime}} .
\end{aligned}
$$

We end up with a Hamiltonian containing cubic, quartic, etc. terms in the phonon creation and annihilation operators. In the space spanned by one- and two-phonon states the bosonic Hamiltonian is

$$
\begin{aligned}
H= & \sum_{\nu} E_{\nu} Q_{\nu}^{\dagger} Q_{\nu}+\left[\sum_{\nu_{1} \nu_{2} \nu_{3}} V_{\nu_{1} \nu_{2} \nu_{3}}^{21} Q_{\nu_{1}}^{\dagger} Q_{\nu_{2}}^{\dagger} Q_{\nu_{3}}^{\dagger}\right. \\
& \left.+\sum_{\nu_{1} \nu_{2} \nu_{3} \nu_{4}} V_{\nu_{1} \nu_{2} \nu_{3} \nu_{4}}^{22} Q_{\nu_{1}}^{\dagger} Q_{\nu_{2}}^{\dagger} Q_{\nu_{3}} Q_{\nu_{4}}\right]+ \text { H.c. },
\end{aligned}
$$

where $V^{21}\left(V^{22}\right)$ are the matrix elements connecting onephoton states with two-phonon states (two-photon states with two-phonon states). The eigenstates of the Hamiltonian (2.5) are

$$
\left|\Phi_{\alpha}\right\rangle=\sum_{\nu} c_{\nu}^{\alpha}|\nu\rangle+\sum_{\nu_{1} \nu_{2}} d_{\nu_{1} \nu_{2}}^{\alpha}\left|\nu_{1} \nu_{2}\right\rangle
$$

and the corresponding eigenvalues do not form a harmonic spectrum.

In the semiclassical models of grazing ion-ion collisions the excitation of one of the two nuclei is due to the mean field of the other. Since the mean field is a one-body operator, the excitation operator has the following form:

$$
W(t)=\sum_{\alpha \beta}\left\langle\alpha\left|U_{B}(\mathbf{R}(t))\right| \beta\right\rangle a_{\alpha}^{\dagger} a_{\beta},
$$

where $U_{B}$ is the mean field of the other nucleus. The time dependence comes in through the relative distance $R$ between the two nuclei. In the standard approach $W(t)$ is linear in the phonon operators because only the ph terms of Eq. (2.7) are considered and the lowest-order boson expansion is taken. If we include also the $p p$ and $h h$ terms, their mapping [Eq. (2.4)] leads to a quadratic form in $Q$

$$
\begin{aligned}
W= & W^{00}+\sum_{\nu} W_{\nu}^{10} Q_{\nu}^{\dagger}+\text { H.c. }+\sum_{\nu \nu^{\prime}} W_{\nu \nu^{\prime}}^{11} Q_{\nu}^{\dagger} Q_{\nu^{\prime}} \\
& +\sum_{\nu \nu^{\prime}} W_{\nu \nu^{\prime}}^{20} Q_{\nu}^{\dagger} Q_{\nu^{\prime}}^{\dagger}+\text { H.c. }
\end{aligned}
$$

The first term in Eq. (2.8) represents the interaction of the two colliding nuclei in their ground state; in the present case it has also an imaginary part that describes the absorption due to the nonelastic channels. The $W^{10}$ part connects states differing by one phonon, the $W^{11}$ term couples excited states with the same number of phonons, while $W^{20}$ allows transitions from the ground state to two-phonon states. All the form factors $W$ are calculated by double folding the Coulomb and nuclear nucleon-nucleon interactions with the HartreeFock ground-state density of the projectile and with the ground state density or the transition densities of the considered excited states of the target.

In the space of the ground state and the $\left|\Phi_{\alpha}\right\rangle$ states we can cast the Schrödinger equation into a set of linear differential coupled equations for the time-dependent amplitude probabilities $A_{\alpha}(t)$. Then the cross section is calculated nonperturbatively as described in Ref. [6] where we integrated the probability of exciting the state $\left|\Phi_{\alpha}\right\rangle$ starting from a minimum impact parameter. In the calculation presented here we integrate over all impact parameters since we have introduced in $W^{00}$ the optical potential, which, in an effective way, takes care of the most inner trajectories.

The imaginary part $W_{\text {im }}$ of the optical potential is usually determined by fitting the experimental elastic cross section. This potential describes the absorption due to all nonelastic channels. Therefore, it cannot be inserted directly in $W^{00}$ [Eq. (2.7)] since the absorption due to the inelastic channels explicitly included in the coupled equations would be counted twice.

Let us first discuss how to solve this problem when no anharmonicities are present and therefore, the states $\left|\Phi_{\alpha}\right\rangle$ are pure multiphonon states. In such a case one can solve the Schrödinger equation in a semiclassical approach by integrating it along each classical relative motion trajectory. The state of the system $|\Psi\rangle$ is a coherent state and the probability to excite $m_{\nu}$ times a phonon $\nu$ is [9]

$$
P_{\nu, m_{\nu}}^{0}=\frac{\left(N_{\nu}\right)^{m_{\nu}}}{m_{\nu} !} P_{\mathrm{g} . \mathrm{s} .}^{0},
$$

where $N_{\nu}$ is the average number of $\nu$ phonons in $|\Psi\rangle$. In the above equation, as well as in the following discussion, the dependence on the impact parameter $b$ is understood. The 
superscript " 0 " refers to the fact that only the absorption due to the multiple excitation of phonons is taken into account. In such a case

$$
P_{\text {g.s. }}^{0}=e^{-\mathcal{N}}
$$

where $\mathcal{N}=\Sigma_{\nu} N_{\nu}$. We stress that the same survival probability of the ground state appears as a factor in all the probabilities in Eq. (2.9).

The survival probability associated with the imaginary optical potential $W_{\text {im }}$ is calculated as

$$
P_{\text {g.s. }}^{W}=\exp \left\{\frac{2}{\hbar c} \int_{-\infty}^{+\infty} W_{\text {im }}(t) d t\right\},
$$

where the integral is again done along a classical trajectory. The depopulation of the ground state due only to the neglected channels can be, in principle, calculated as in Eq. (2.11) but with an auxiliary imaginary potential $\bar{W}$, which does not contain the absorption due to the adopted ones. Then

$$
P_{\mathrm{g} . \mathrm{s} .}^{W}=P_{\mathrm{g} . \mathrm{s} .}^{0} \times P_{\mathrm{g} . \mathrm{S} .}^{\bar{W}}
$$

and

$$
P_{\nu, m_{\nu}}=P_{\nu, m_{\nu}}^{0} \times P_{\text {g.s. }}^{\bar{W}} .
$$

When anharmonicities are taken into account, the state of the system is no more a coherent state. The probability to excite the state $\left|\Phi_{\alpha}\right\rangle$ is equal to

$$
P_{\alpha}^{0}=\left|A_{\alpha}\right|^{2}
$$

where $A_{\alpha}$ is solution of the coupled equations of motion without any imaginary potential. Therefore, $P_{\alpha}^{0}$ contains only the absorption due to all the adopted channels. The remaining part, due to all the other channels, can be introduced by writing, in analogy with Eq. (2.13),

$$
P_{\alpha}=P_{\alpha}^{0} \times P_{\text {g.s. }}^{\bar{W}} \text {. }
$$

This equation can be formally derived by assuming that the absorption due to the excluded channels is the same in all the adopted ones. This is certainly an approximation, however, we would like to emphasize that many important inelastic channels are explicitly taken into account in the coupled equations and that we solve the latter exactly. Therefore, the corresponding absorption is calculated correctly, including the $Q$-value effects. The unknown auxiliary imaginary potential $\bar{W}$ can be eliminated by inserting Eq. (2.12) in Eq. (2.15),

$$
P_{\alpha}=P_{\alpha}^{0} \times \frac{P_{\text {g.s. }}^{W},}{P_{\text {g.s. }}^{0}},
$$

which is the expression we have used in order to calculate the inelastic cross section. We would like to stress that the part of the nuclear absorption that corresponds to noninelastic channels is often taken into account as a sharp cutoff
TABLE I. RPA one-phonon basis for the nucleus ${ }^{40} \mathrm{Ca}$. We have two giant monopole resonance (GMR) states as well as two dipole (GDR) and two quadrupole: isoscalar (ISGQR) and isovector (IVGQR). For the octupole resonance we have the low energy (LEOR) and the high energy one (HEOR). For each state its spin, parity, isospin, energy, and percentage of the EWSR are reported.

\begin{tabular}{lllcc}
\hline \hline \multicolumn{1}{c}{ Phonons } & $J^{\pi}$ & $T$ & $E(\mathrm{MeV})$ & EWSR $(\%)$ \\
\hline GMR $_{1}$ & $0^{+}$ & 0 & 18.25 & 30 \\
GMR $_{2}$ & $0^{+}$ & 0 & 22.47 & 54 \\
GDR $_{1}$ & $1^{-}$ & 1 & 17.78 & 56 \\
GDR $_{2}$ & $1^{-}$ & 1 & 22.03 & 10 \\
ISGQR & $2^{+}$ & 0 & 16.91 & 85 \\
IVGQR $_{3}^{-}$ & $2^{+}$ & 1 & 29.59 & 26 \\
$3^{-}$ & $3^{-}$ & 0 & 4.94 & 14 \\
HEOR & $3^{-}$ & 0 & 9.71 & 5 \\
\hline \hline
\end{tabular}

transmission coefficient. So the introduction of the imaginary potential can be seen as an important improvement.

\section{RESULTS AND DISCUSSION}

The above described model has been applied to the reaction ${ }^{40} \mathrm{Ca}$ on ${ }^{40} \mathrm{Ca}$ at $E /$ nucleon $=50 \mathrm{MeV}$. The one-phonon basis has been obtained with a self-consistent HF+RPA calculation with Skyrme interaction SGII [10]. Only the most collective one-phonon states, exhausting at least $5 \%$ of the relevant energy-weighted sum rule (EWSR), are taken into account. They are listed in Table I. We then have considered all possible two-phonon states that can be constructed out of them, with all possible values of the total angular momentum $L$, and in this space we have diagonalized the Hamiltonian (2.5) to get the states (2.6). In Table II we have reported some properties of the quadrupole states, each one labeled with the name of its main component and whose unperturbed energy is given in the second column. In the third column there are the energy shifts due to the anharmonicities. Their overlaps with the single and double isoscalar giant quadrupole resonance (ISGQR) states are shown in the last two columns. Similar tables for the DGR states are reported in [6].

The elementary nuclear form factors $W$ for pure one- and two-phonon configurations [Eq. (2.8)] were calculated by double folding the M3Y nucleon-nucleon interaction [11] with the RPA transition densities. The transition matrix elements between mixed states $\left|\Phi_{\alpha}\right\rangle$ were computed by mixing the elementary form factors according to the unitary transformation (2.6). The same procedure was used with the Coulomb interaction to calculate the Coulomb form factors. The relative motion trajectories were determined by solving the classical equation of motion in the presence of both the Coulomb field and the real part of the nuclear potential.

The real part of the optical potential was obtained by double folding the M3Y nucleon-nucleon potential with the Hartree-Fock densities of the two nuclei while its imaginary part was chosen with the same geometry and multiplied by a scale factor whose value (0.627) was determined by a fit to the experimental elastic cross section for the collision ${ }^{40} \mathrm{Ca}$ 
TABLE II. Characteristics of the $\left|\Phi_{\alpha}\right\rangle$ quadrupole $2^{+}$states whose major components are in the first column. In the second column we show the energies of the major components in the harmonic approach. The shift in the energy produced by the anharmonicities is indicated by $\Delta E$ (in $\mathrm{KeV}$ ). We can compare these values with the diagonal matrix elements of the residual interaction, $\Delta E_{0}$ (in $\mathrm{KeV}$ ). In the last columns we report the amplitude with which the single and double ISGQR components appear in the mixed states.

\begin{tabular}{|c|c|c|c|c|c|c|c|}
\hline Quadrupole & & States & $E_{0}(\mathrm{MeV})$ & $\Delta E$ & $\left(\Delta E_{0}\right)$ & $c_{\text {ISGQR }}$ & $c_{\text {ISGQR-ISGQR }}$ \\
\hline ISGQR & & & 16.910 & -402 & 0 & 0.985 & -0.014 \\
\hline IVGQR & & & 29.594 & -506 & 0 & -0.005 & 0.017 \\
\hline $\mathrm{GMR}_{1}$ & $\otimes$ & ISGQR & 35.155 & 87 & -11 & -0.073 & -0.028 \\
\hline $\mathrm{GMR}_{1}$ & $\otimes$ & IVGQR & 47.845 & -42 & -187 & -0.000 & 0.002 \\
\hline $\mathrm{GMR}_{2}$ & $\otimes$ & ISGQR & 39.378 & 246 & -31 & -0.108 & -0.014 \\
\hline $\mathrm{GMR}_{2}$ & $\otimes$ & IVGQR & 52.067 & 190 & -178 & -0.002 & 0.003 \\
\hline $\mathrm{GDR}_{1}$ & $\otimes$ & $\mathrm{GDR}_{1}$ & 35.560 & -464 & -505 & 0.034 & 0.087 \\
\hline $\mathrm{GDR}_{1}$ & $\otimes$ & $\mathrm{GDR}_{2}$ & 39.814 & -436 & -439 & 0.009 & 0.006 \\
\hline $\mathrm{GDR}_{1}$ & $\otimes$ & $3^{-}$ & 22.722 & -31 & -35 & 0.029 & -0.000 \\
\hline $\mathrm{GDR}_{1}$ & $\otimes$ & LEOR & 27.486 & -444 & -442 & -0.013 & -0.007 \\
\hline $\mathrm{GDR}_{1}$ & $\otimes$ & HEOR & 49.110 & -278 & -288 & -0.005 & 0.006 \\
\hline $\mathrm{GDR}_{2}$ & $\otimes$ & $\mathrm{GDR}_{2}$ & 44.058 & -435 & -436 & 0.004 & 0.002 \\
\hline $\mathrm{GDR}_{2}$ & $\otimes$ & $3^{-}$ & 26.976 & -6 & 7 & 0.003 & 0.001 \\
\hline $\mathrm{GDR}_{2}$ & $\otimes$ & LEOR & 31.740 & -307 & -309 & 0.600 & -0.007 \\
\hline $\mathrm{GDR}_{2}$ & $\otimes$ & HEOR & 53.364 & -212 & -217 & 0.000 & 0.000 \\
\hline ISGQR & $\otimes$ & ISGQR & 33.819 & 0 & 4 & -0.020 & 0.995 \\
\hline ISGQR & $\otimes$ & IVGQR & 46.508 & 39 & 40 & 0.002 & 0.002 \\
\hline IVGQR & $\otimes$ & IVGQR & 59.198 & -247 & -250 & -0.007 & -0.004 \\
\hline $3^{-}$ & $\otimes$ & $3^{-}$ & 9.884 & 750 & 776 & -0.045 & -0.005 \\
\hline $3^{-}$ & $\otimes$ & LEOR & 14.648 & -267 & -241 & 0.086 & 0.001 \\
\hline $3^{-}$ & $\otimes$ & HEOR & 36.272 & -104 & -120 & 0.025 & -0.003 \\
\hline LEOR & $\otimes$ & LEOR & 19.413 & -271 & -269 & -0.021 & -0.000 \\
\hline LEOR & $\otimes$ & HEOR & 41.037 & -192 & -197 & -0.005 & 0.002 \\
\hline HEOR & $\otimes$ & HEOR & 62.660 & -212 & -215 & -0.006 & -0.001 \\
\hline
\end{tabular}

on ${ }^{40} \mathrm{Ca}$ at $\mathrm{E} /$ nucleon $=50 \mathrm{MeV}$ of Ref. [12].

In these calculations both the nuclear and Coulomb excitations were included. Actually, the Coulomb excitation alone does not produce a sizable cross section because the colliding nuclei are not very heavy, but when it is considered together with the nuclear excitation it produces an interference effect, which can be important. This is due to the fact that on one hand we have a coupled channel effect and, on the other hand, some two-phonon states are excited only when both fields are acting. This was clearly demonstrated in our previous work [7].

Since our calculations are based on a discrete RPA we get a discrete excitation spectrum and a cross section $\sigma_{\alpha}$ corresponding to each state $\left|\Phi_{\alpha}\right\rangle$. The energy differential cross sections presented in Fig. 1 are obtained by summing up all the contributions coming from the states $\left|\Phi_{\alpha}\right\rangle$ after a smoothing of each individual line by a Lorentzian with a 3 $\mathrm{MeV}$ width. The dashed line refers to a calculation where the internal Hamiltonian is harmonic and the external field is linear. The solid line corresponds to a calculation where the anharmonicity and nonlinearity were introduced, which produce a sizable increase with respect to the standard case. In the figure we can clearly distinguish three energy regions. The cross sections given in Tables III-V are obtained by summing up the $\sigma_{\alpha}$ 's for the discrete states $\left|\Phi_{\alpha}\right\rangle$ lying in each region. As already observed in Refs. [6,7], the increase at low energies is due both to the anharmonicities and nonlinearities. In particular, the anharmonicities are important because the low-lying two-phonon states can be excited by the $W^{10}$ part of the external field through their large onephonon component. At high energies the main contribution comes from the nonlinearities because their presence increases the number of excitation routes. This is seen better in Table IV where the excitation cross section in the double giant quadrupole resonance energy region is reported. For each multipolarity we have summed the excitation cross section in the energy region between 28 and $38 \mathrm{MeV}$, and this is done for four different cases as shown in the table. The $L$ $=3$ contribution is due to the HEOR at $31.33 \mathrm{MeV}$, while the $L=0,2$, and 4 contributions are dominated by the double excitation of the double ISGQR. As we can see in Table V, the nonlinear terms are also responsible for the increase of the cross section in the ISGQR region, especially for the $L$ $=2$ state whose main component is the ISGQR. This is at variance with the relativistic Coulomb excitation studied in Ref. [6] because the Coulomb interaction very selectively populates dipole transitions and therefore cannot excite the most important two-phonon components of the ISGQR, which are built with monopole and quadrupole phonons (see Table II).

The obtained ratio between the cross section in the giant 


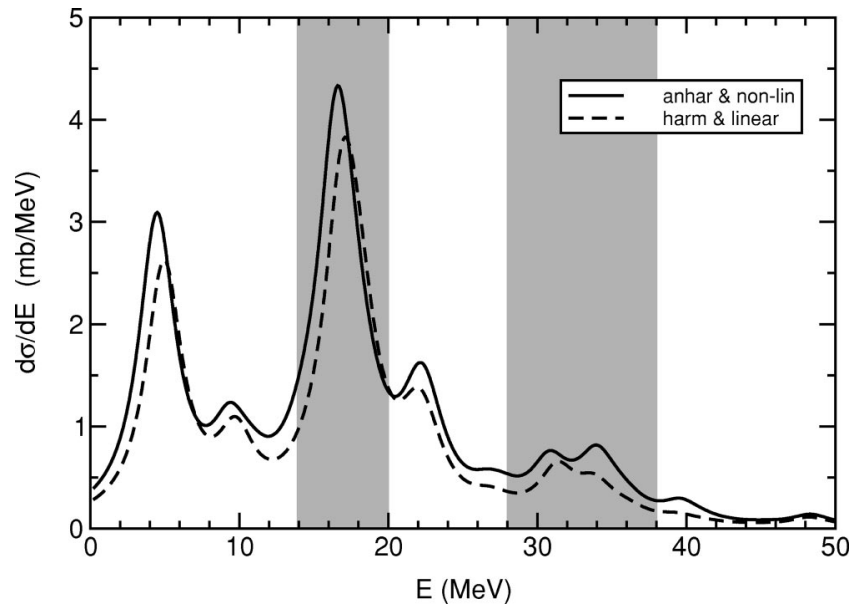

FIG. 1. Inelastic cross section for the system ${ }^{40} \mathrm{Ca}+{ }^{40} \mathrm{Ca}$ at 50 $\mathrm{MeV} /$ nucleon as a function of the excitation energy. Both curves are the result of a smoothing procedure with a Lorentzian of width $\Gamma$ $=3 \mathrm{MeV}$. The shadowed areas are the energy regions over which we have summed the cross sections reported in the tables.

resonance region and that in the two-phonon region varies from 3.7 in the anharmonic and nonlinear case to 4.6 in the harmonic and linear calculation. If we only consider the cross section up to the single and double isoscalar giant quadrupole resonance, these ratios increase to 6.5 and 9.6, respectively. Those values are smaller than the ones reported in Ref. [3] for the cross sections at the grazing angle. This difference can be traced back to the present availability of the experimental elastic cross section needed to fix the imaginary part of the optical potential and to the fact that the theoretical approach has been improved in several aspects, especially in the calculation of the form factors.

Our calculation can be compared with the experimental data of Ref. [4] where the reaction ${ }^{40} \mathrm{Ca}+{ }^{40} \mathrm{Ca}$ at $50 \mathrm{MeV} /$ nucleon has been studied. Let us resume the important results of Ref. [4] and the most critical points. We discuss first the inclusive spectrum and later on we will analyze the one obtained in coincidence with backward emitted particles. The inelastic spectrum was extracted for ejectiles scattered between $3.4^{\circ}$ and $10^{\circ}$ in the center of mass frame. The GR

TABLE III. Coulomb plus nuclear excitation cross section for ${ }^{40} \mathrm{Ca}+{ }^{40} \mathrm{Ca}$ at $50 \mathrm{MeV} /$ nucleon. Each multipolarity contribution is shown for several anharmonic and nonlinear combinations. The values for $L=1$ and 5 are very small and they are not shown. The cross sections (in millibarns) are summed over the energy region $(0 \leqslant E \leqslant 12 \mathrm{MeV})$.

\begin{tabular}{ccccc}
\hline \hline Phonons & $\begin{array}{c}\text { Harm. } \\
\text { and lin. }\end{array}$ & $\begin{array}{c}\text { Harm. } \\
\text { and nonlin. }\end{array}$ & $\begin{array}{c}\text { Anh. } \\
\text { and lin. }\end{array}$ & $\begin{array}{c}\text { Anh. } \\
\text { and nonlin. }\end{array}$ \\
\hline$L=0$ & 0.1 & 0.3 & 1.3 & 2.3 \\
$L=2$ & 0.2 & 0.4 & 0.2 & 0.1 \\
$L=3$ & 14.2 & 16.9 & 14.3 & 16.8 \\
$L=4$ & 0.2 & 0.3 & 0.2 & 0.3 \\
$L=6$ & 0.5 & 0.7 & 0.4 & 0.7 \\
Total & 15.2 & 18.6 & 16.4 & 20.2 \\
\hline \hline
\end{tabular}

TABLE IV. Same as Table III but for the double ISGQR region. The cross sections (in millibarns) are summed over the energy region $(28 \leqslant E \leqslant 38 \mathrm{MeV})$. The values in parentheses correspond to the double ISGQR state.

\begin{tabular}{cllll}
\hline \hline Phonons & $\begin{array}{c}\text { Harm. } \\
\text { and lin. }\end{array}$ & $\begin{array}{c}\text { Harm. } \\
\text { and nonlin. }\end{array}$ & $\begin{array}{c}\text { Anh. } \\
\text { and lin. }\end{array}$ & $\begin{array}{c}\text { Anh. } \\
\text { and nonlin. }\end{array}$ \\
\hline$L=0$ & $0.2(0.15)$ & $0.3(0.26)$ & $0.2(0.14)$ & $0.3(0.21)$ \\
$L=2$ & $0.6(0.33)$ & $1.0(0.51)$ & $0.6(0.33)$ & $1.1(0.53)$ \\
$L=3$ & 2.2 & 2.5 & 2.3 & 2.5 \\
$L=4$ & $1.0(0.90)$ & $1.9(1.83)$ & $0.9(0.85)$ & $1.8(1.73)$ \\
$L=6$ & 0.2 & 0.2 & 0.2 & 0.2 \\
Total & $4.2(1.38)$ & $5.9(2.60)$ & $4.2(1.32)$ & $5.9(2.47)$ \\
\hline \hline
\end{tabular}

contribution was obtained from the inclusive inelastic spectrum by deconvolution of the angular distributions into inelastic excitations and a noninelastic background. For the inelastic excitation, a distorted-wave Born approximation (DWBA) prediction was used. As for the background, its angular distribution was assumed to be similar to the one of the energy region located immediately above the GRs. This procedure gave $113 \mathrm{mb} / \mathrm{sr}$ between 12 and $22 \mathrm{MeV}$ for the inelastic excitation corresponding to $40 \%$ of the quadrupole EWSR. However, it should be noticed that the estimate of the noninelastic background underlying the GR is not unambiguous. Indeed, if inelastic excitation is still present in the region above the resonance as expected from Fig. 1, the assumed background is overestimated. In this case the extracted value should be understood as a minimum. The maximum inelastic contribution compatible with the measured angular distribution is $223 \mathrm{mb} / \mathrm{sr}$. This corresponds to the other extreme when no noninelastic background is considered. Therefore the GR cross section extracted from the inclusive spectrum is between 113 and $223 \mathrm{mb} / \mathrm{sr}$ depending upon the background hypothesis. The associated EWSR would thus range between $40 \%$ and $80 \%$ if the whole cross section is assumed to be coming from quadrupole states.

In order to get the total cross section one has to extrapolate the measured differential cross section beyond the solid angle covered by the ejectile detector. This was done by assuming that the DWBA angular distribution used to fit the measured angular distribution in Ref. [4] was also valid in

TABLE V. Same as Table III but for the ISGQR region. The cross sections (in millibarns) are summed over the energy region $(14 \leqslant E \leqslant 20 \mathrm{MeV})$. In this region there are no states with $L=3$ and 5. The values in parentheses correspond to the ISGQR state.

\begin{tabular}{ccccc}
\hline \hline Phonons & $\begin{array}{c}\text { Harm. } \\
\text { and lin. }\end{array}$ & $\begin{array}{c}\text { Harm. } \\
\text { and nonlin. }\end{array}$ & $\begin{array}{c}\text { Anh. } \\
\text { and lin. }\end{array}$ & $\begin{array}{c}\text { Anh. } \\
\text { and nonlin. }\end{array}$ \\
\hline$L=0$ & 2.7 & 2.8 & 2.2 & 2.2 \\
$L=1$ & 3.0 & 2.9 & 3.6 & 3.3 \\
$L=2$ & $13.3(13.2)$ & $16.1(16.0)$ & $13.8(13.6)$ & $16.0(16.0)$ \\
$L=4$ & 0.1 & 0.1 & 0.1 & 0.1 \\
$L=6$ & 0.2 & 0.3 & 0.2 & 0.3 \\
Total & 19.3 & 22.2 & 19.9 & 21.9 \\
\hline \hline
\end{tabular}


the region in which no data are available. The ratio between the integrals of the DWBA cross section over the full angular range and that over the angles covered by the detector is 3.16. Taking into account the fraction of the solid angle covered by the spectrometer one gets a total compensating factor of $6.67 \times 10^{-2}$. Such a factor transforms the double differential cross section into the energy differential one. The resulting total cross section is then 7.5 and $15 \mathrm{mb}$, respectively. These values have to be compared with the theoretical inelastic cross section, which, in the anharmonic and nonlinear case, adds up to $22 \mathrm{mb}$ in the GR region. Taking into account the uncertainties of the analysis of the experimental data and the fact that our theoretical results are obtained without adjusting any parameter, the comparison can be considered satisfactory. In order to draw quantitative conclusions one should elaborate on different issues both from the experimental and theoretical sides. A recent experiment on the same reaction [13] using an improved apparatus is expected to eliminate most of the experimental uncertainties. These new data will allow a more reliable determination of some parameters entering in the theoretical calculation, mainly in the optical potential.

Coincidences with backward emitted particles provide an unambiguous signal for the inelastic excitations and could, in principle, be used to avoid the noninelastic background problems. This was the idea of Ref. [4], but some other sources of uncertainties appear. The coincidence rate with backward emitted protons was converted into a differential cross section, correcting for the energy dependence of the proton multiplicity. At that time it was already stressed that this correction factor can be subject to many uncertainties. First of all, this proton multiplicity function was calculated with a statistical decay code, which does not include any direct decay component. Furthermore, due to the absence of out-of-plane detectors, the azimuthal angular distribution was not measured and was assumed to be uniform. This procedure gives a cross section for the GR extracted from the coincidence data $(339 \mathrm{mb} / \mathrm{sr})$ larger than the one obtained from the inclusive inelastic spectrum (between 113 and $223 \mathrm{mb} / \mathrm{sr}$ ). This shows that the hypotheses used are not correct. The use of a $4 \pi$ detector in a recent experiment [13] should solve these ambiguities since it will provide the angular distribution of the emitted protons and there will be no need to rely on a statistical code to infer their multiplicity. That was not the case in the experiment of Ref. [4]. Therefore, only the ratio was deduced from the coincidence data. Two values of this ratio were reported by assuming two backgrounds for the two-phonon region, while the GR peak was considered with no background subtraction in the coincidence spectrum. The values of the second phonon cross section were, after subtraction of the two backgrounds, 30 and $17 \mathrm{mb} / \mathrm{sr}$, respectively, for an energy running from 28 to $40 \mathrm{MeV}$, while the GR cross section was $339 \mathrm{mb} / \mathrm{sr}$ in the coincidence spectrum in the range $12-22 \mathrm{MeV}$, leading to the ratios 11 and 20 quoted in Ref. [4]. ${ }^{1}$ Such values are the ratios between the

\footnotetext{
${ }^{1}$ One digit-inversion error was spotted in the text of Figs. 16(c) and 16(d) of Ref. [4] (erratum to be published).
}

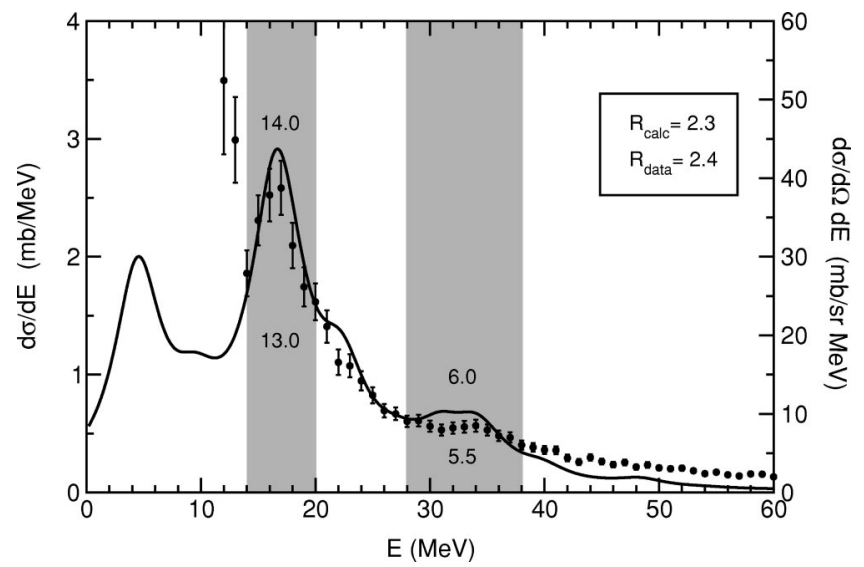

FIG. 2. The dots represent the experimental coincidence inelastic spectrum of Ref. [4] [Fig. 16(b)] with no background subtraction (right scale). The solid line is the result of a smoothing procedure with a Lorentzian with a width $\Gamma=5 \mathrm{MeV}$ of the theoretical inelastic cross section for the anharmonic and nonlinear case (left scale). The shadowed areas are the energy regions over which we have integrated the energy differential cross sections. The resulting values in millibarns are the numbers reported in the two areas. Those above the curves refer to the theoretical results, while the ones below refer to the experimental data. In the inset we report the ratios between the single GR cross section and the DGR ones for the two cases.

single GR cross section and only a small fraction of the DGR cross section. We want to stress here that the correct procedure should be not to subtract any background in the twophonon region. Indeed, on one hand, coincidence with backward emitted particles avoids any contribution from noninelastic background in the experimental data. On the other hand, in our theoretical calculation, not only double GQR has been included but many contributions from different inelastic excitations have been taken into account. These two remarks plead in favor of a direct comparison of the oneand the two-phonon regions with no background subtraction.

In order to have a more direct comparison we present, in Fig. 2, the experimental coincidence inelastic spectrum of Ref. [4] [Fig. 16(b)] with no background subtraction. The right scale is the double differential cross section while the left scale is the energy differential cross section obtained with the above mentioned factor of $6.67 \times 10^{-2}$. In the figure we present the theoretical results smoothed by a Lorentzian of $5 \mathrm{MeV}$ width, rather than the $3 \mathrm{MeV}$ used in Fig. 1. From the figure we see that with this value the shape of the experimental peak in the GR region is well reproduced. It should be noticed that some contribution to the experimental cross section is present just below $14 \mathrm{MeV}$. However, due to the proximity of the proton emission threshold, the correction for the multiplicity is more delicate in that energy region. Disregarding these two points, the overall agreement between theory and experiment is rather satisfactory. A rough estimate of the one-phonon and two-phonon cross sections can be obtained by integrating both curves in the energy ranges shown in Fig. 2 as shadowed areas. By doing that one would get an experimental and theoretical ratio of 2.4 and 2.3, respectively. We want to stress that the experimental ratio 
quoted above is different from the one deduced in Ref. [4] because the latter is the ratio between the full peak of the single GR and the DGR with background subtraction, while the former one is obtained without background subtraction in both single GR and DGR. Furthermore, the first two experimental points in Fig. 2 were not included as explained before. Finally, we would like to comment on the dependence of the theoretical ratios upon the smoothing width. This ratio is decreasing with the increasing width, due to the fact that while the integral of the single GR is decreasing, that over the region of the DGR remains almost unchanged. This is related to the fact that in the single GR energy region the peaks of the single $\Phi_{\alpha}$ states are quite separate while the density of states in the DGR region is very high. In any case the dependence on the width is not very strong; by varying $\Gamma$ from 3 to $6 \mathrm{MeV}$ the ratio changes from 2.75 to 2.20 . These values cannot be directly compared with the values reported in Tables III-V because the latter have been obtained just by summing the cross sections associated with each discrete state.

\section{CONCLUSIONS}

We have calculated the inelastic scattering cross sections of one- and two-phonon states for the ${ }^{40} \mathrm{Ca}+{ }^{40} \mathrm{Ca}$ collision at $E /$ nucleon $=50 \mathrm{MeV}$. Several effects have been evidenced. In particular, we have analyzed the role played by anharmonicities in the excitation spectrum and nonlinearities in the operator describing the mutual interaction of the collision partners. The anharmonicities are particularly important at relatively low energy where the excitation comes through the one-phonon component of the mixed states. The nonlineari- ties give their main contribution at high energy, in particular, in the region of the double quadrupole giant resonance. Namely, in the interval between 28 and $38 \mathrm{MeV}$, they give an increase of about $40 \%$ with respect to a harmonic and linear calculation. This increase is due to the excitation of other two-phonon states that are populated because of the presence of the anharmonicities and nonlinearities. With all the previously discussed caveats, the comparison of the smoothed theoretical result with the experimental coincidence inelastic spectrum of Ref. [4] is satisfactory. The inclusion of threephonon states in the calculation will increase the inelastic cross section at higher excitation energies. At the same time, a fraction of the population of the two-phonon states will move to higher energies. In Ref. [14] it has been shown, within a simple model, that the spectrum calculated by diagonalizing the Hamiltonian obtained by a boson expansion truncated at the quartic order in a space including up to three phonons is in reasonable agreement with the exact one. A similar calculation is feasible also in a realistic case. This, together with the results shown here, encourages us to proceed in the direction of calculating the three-phonon excitation cross section for the system ${ }^{40} \mathrm{Ca}+{ }^{40} \mathrm{Ca}$ at $E$ /nucleon $=50 \mathrm{MeV}$ for which experiments have already been done [13].

\section{ACKNOWLEDGMENTS}

This work has been partially supported by the Spanish DGICyT under Contract No. PB98-1111, by the SpanishItalian agreement between the CICyT and the INFN, and by the Spanish-French agreement between the CICyT and the IN2P3.
[1] H. Emling, Prog. Part. Nucl. Phys. 33, 729 (1994); Ph. Chomaz and N. Frascaria, Phys. Rep. 252, 275 (1995); T. Aumann, P. F. Bortignon, and H. Emling, Annu. Rev. Nucl. Part. Sci. 48, 351 (1998).

[2] C. Benesh, B. Cook, and J. Vary, Phys. Rev. C 40, 1198 (1989).

[3] F. Catara, Ph. Chomaz, and A. Vitturi, Nucl. Phys. A471, 661 (1987).

[4] J. A. Scarpaci et al., Phys. Rev. C 56, 3187 (1997); Phys. Rev. Lett. 71, 3766 (1993).

[5] C. Volpe, F. Catara, Ph. Chomaz, M. V. Andrés, and E. G. Lanza, Nucl. Phys. A589, 521 (1995); A599, 347c (1996).

[6] E. G. Lanza, M. V. Andrés, F. Catara, Ph. Chomaz, and C. Volpe, Nucl. Phys. A613, 445 (1997); A654, 792c (1999).
[7] E. G. Lanza, M. V. Andrés, F. Catara, Ph. Chomaz, and C. Volpe, Nucl. Phys. A636, 452 (1998).

[8] M. Hage-Hassan and M. Lambert, Nucl. Phys. A188, 545 (1972).

[9] K. Alder and A. Winther, Electromagnetic Excitation (NorthHolland, Amsterdam, 1975).

[10] N. V. Giai and H. Sagawa, Phys. Lett. 106B, 379 (1981).

[11] G. R. Satchler and W. G. Love, Phys. Rep. 55, 183 (1979).

[12] J. A. Scarpaci, Ph.D. thesis, Université d'Orsay, France, 1990.

[13] N. Frascaria, Nucl. Phys. A687, 154 (2001); (private communication).

[14] C. Volpe, Ph. Chomaz, M. V. Andrés, F. Catara, and E. G. Lanza, Nucl. Phys. A647, 246 (1999). 